\title{
Special Issue on the 3rd International Conference Microelectronics, Circuits and Systems (Micro 2016)
}

\author{
Jyotsna Kumar Mandal $^{1} \cdot$ Abhijit Biswas $^{2} \cdot$ Dulal Acharjee $^{3}$
}

Published online: 11 April 2019

(C) Springer-Verlag GmbH Germany, part of Springer Nature 2019

The 3rd International Conference on Microelectronics, Circuits and Systems (Micro 2016) was held in Kolkata, India during July 9-10, 2016 in which 120 articles were presented. Out of these presentations extended version of only 31 promising papers were selected for submission to the Microsystem Technologies (MST). Authors of these selected papers were intimated to enlarge their manuscript by adding at least $50 \%$ more research finding in the relevant topic compared to that presented in Micro2016 along with the change in title. In addition, it was advised to compare the results presented in the revised articles with the state-of-the-art results available in the recent literature to justify improvements. The modifications were first checked by the Guest Editors and then the papers were uploaded by the authors to the portal of MST. The articles were reviewed by at least two blind reviewers as per the norms of the Microsystem Technologies. On the basis of reviewers' comments the articles were revised by the authors, and then the revised papers were re-submitted. The revised papers were reexamined by the Guest Editors, and in some cases by the reviewers as well, and then the final publication decision on the paper was made. In this process 24 research articles are finally selected for publication in this special issue.

On behalf of the organizing committee of Micro2016, we would like to express our sincere thanks to the authors for submitting extended version of their articles into this special issue. We express our sincere gratitude to the reviewers for reviewing the articles. We appreciate the effort of the Editor-in-Chief of Microsystem Technologies for providing us the opportunity to publish the extended and revised version of some deserving papers of Micro2016 in the Microsystem Technologies.

Hope this special issue will convey valuable research information to the readers.

Guest Editors

Jyotsna Kumar Mandal

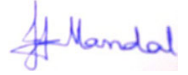

Abhijit Biswas

Min

Dulal Acharjee

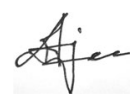

Publisher's Note Springer Nature remains neutral with regard to jurisdictional claims in published maps and institutional affiliations.

Jyotsna Kumar Mandal

jkm.cse@gmail.com

University of Kalyani, Kalyani, West Bengal, India

2 University of Calcutta, Kolkata, West Bengal, India

3 Applied Computer Technology, Kolkata, West Bengal, India 